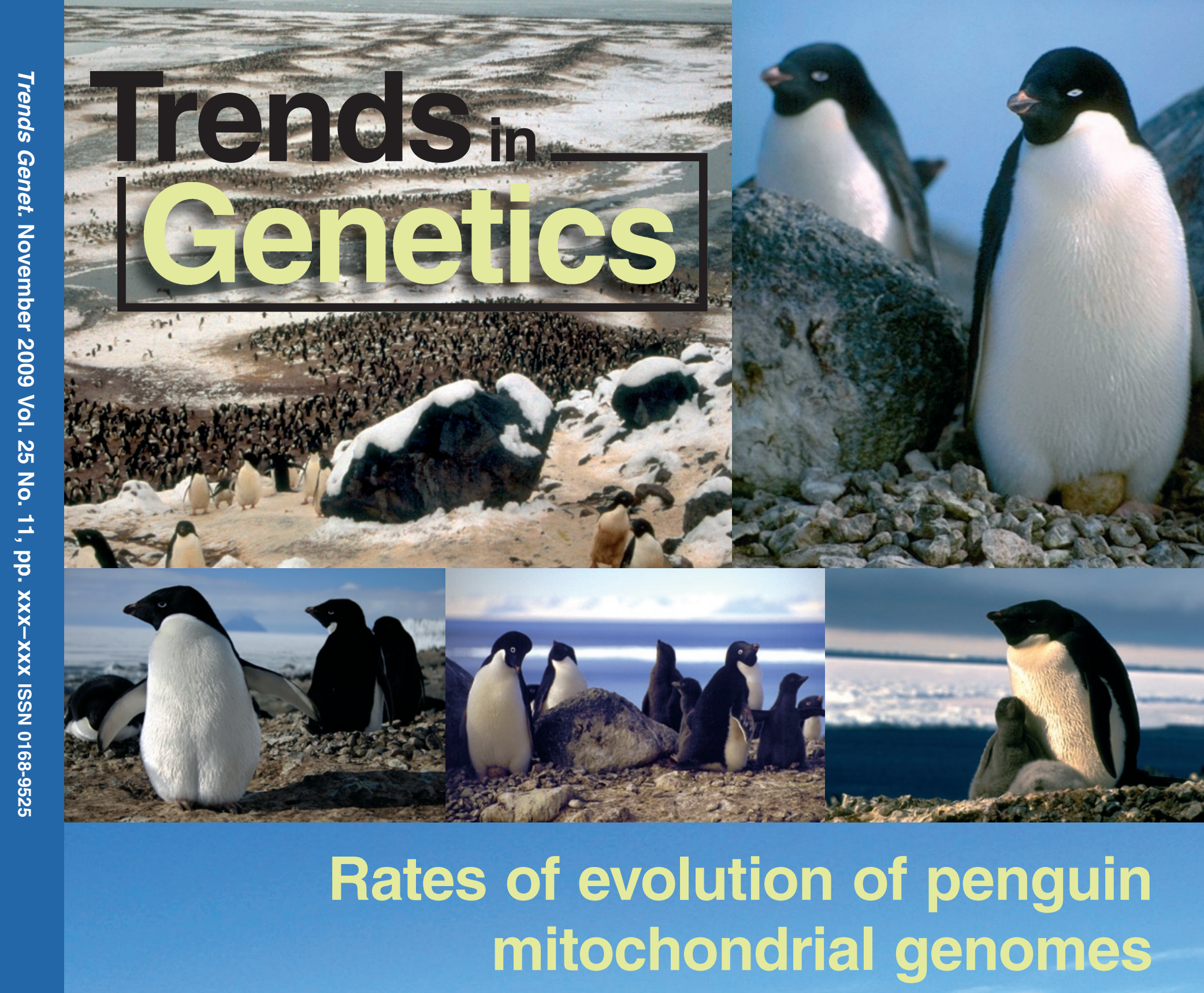

Racial disparities in human genomics How many pathways result in microcephaly?

Chromatin indexing in Arabidopsis

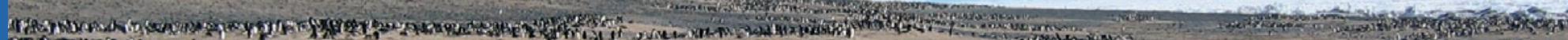

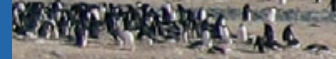

(A) 
2 High mitogenomic evolutionary rates and time dependency

3

Sankar Subramanian ${ }^{1,2}$, Dee R. Denver ${ }^{3,4}$, Craig D. Millar ${ }^{2}$, Tim Heupink ${ }^{3}$, Angelique Aschrafi $^{3,5}$, Steven D. Emslie ${ }^{6}$ Carlo Baroni ${ }^{7}$ and David M. Lambert ${ }^{1,3 *}$

${ }^{1}$ Griffith School of Environment and the School of Biomolecular and Physical Sciences, Griffith University, 170 Kessels Road, Nathan, Qld 4111 Australia.

${ }^{2}$ Allan Wilson Centre for Molecular Ecology and Evolution, School of Biological Sciences, University of Auckland, Private Bag 92019, Auckland, New Zealand.

${ }^{3}$ Allan Wilson Centre for Molecular Ecology and Evolution, Institute of Molecular BioSciences, Massey University, Auckland, New Zealand.

${ }^{4}$ Department of Zoology and Center for Genome Research and Biocomputing, Oregon State University, Corvallis, Oregon, USA.

${ }^{5}$ Department of Cell Biology, The Scripps Research Institute, La Jolla, California 92037, USA.

${ }^{6}$ Department of Biology and Marine Biology, University of North Carolina, Willmington, North Carolina, USA.

${ }^{7}$ Dipartmento Scienze della Terra, Universita` di Pisa, and Consiglio Nazionale Ricerche, Centro Studio Geologia Strutturale, Pisa, Italy.

Using entire modern and ancient mitochondrial genomes of Adélie penguins (Pygoscelis adeliae) that are up to 44,000 years old, we show that mitogenomic rates of evolution are two to six times higher than phylogenetic rate estimates. Although the rate at constrained sites including nonsynonymous positions and RNAs varies over two fold with time (between shallow and deep nodes), the rate of synonymous site evolution remains the same. The time independent neutral evolutionary rates reported here will be useful for the study of recent evolutionary events.

Corresponding author: Lambert, D.M. (d.lambert@griffith.edu.au). 


\section{Rates of molecular evolution}

2 Rates of molecular evolution underpin much of modern evolutionary biology because

3 they can be used, for example, to estimate the time of divergence between

4 species/lineages and to estimate the age of morphological novelties. Generally

5 molecular rates have been estimated using phylogenetic comparisons among multiple

6 species, where levels of divergence have been calibrated using points in the fossil

7 record [1-4] or major biogeographic events [5]. Recent studies have shown a much

8 higher rate of evolution within species compared to the rate obtained using inter-

9 species comparisons [6, 7]. Based on these observation it has been suggested that

10 rates of molecular evolution are time dependent $[8,9]$. The apparent time dependency

11 of evolutionary rates is potentially a result of two factors. First, the estimation of

12 inter-species divergence at neutral sites such as synonymous positions and the hyper-

13 variable region is likely to suffer from the saturation effects due to multiple

14 substitutions. This would result in an underestimation of molecular rates over long

15 time periods [10-12]. Second, the presence of short-lived slightly deleterious

16 mutations among the populations of a species would lead to an overestimation of the

17 actual substitutions over shorter time scales [12-14]. Although the rate estimations

18 based on within species comparisons are not influenced by saturation effects, the 19 presence of short-lived deleterious mutations still poses an obstacle to obtaining 20 unbiased estimates of evolutionary rates for single species. Furthermore, 21 phylogenetic methods cannot be used to infer intra-species rates due to the lack of 22 calibration points within a short timescale.

23 We have used serially preserved ancient DNA samples to resolve this impasse by 24 sequencing the mitochondrial genomes of 12 modern and eight ancient Adélie 25 penguins from Antarctica, including two samples dating back 44,000 years [7, 15]. 
1 These sequences enabled us to estimate precisely the overall mitochondrial genomic

2 evolutionary rate and specific rates for ribosomal RNAs, transfer RNAs, synonymous

3 and nonsynonymous positions of the protein-coding regions. These specific rates

4 enabled us to examine the effects of time dependency on rates of molecular evolution.

5 We have used three different analytical methods to estimate these molecular rates in 6 mitochondrial genomes of Adélie penguins.

8 Estimating genomic rates of evolution using ancient mitochondrial DNA

9 We amplified and sequenced mitochondrial genomes using blood samples of modern 10 Adélie penguins and from the sub-fossil bones of these birds from Antarctica (Figure

11 1). The size of the genome recovered from each of these ancient samples was $12.5-$

$1216.1 \mathrm{~kb}$ (Figure S1). The ages of these sub-fossil bones ranged from 250 to 44,000

13 years, which were estimated using radiocarbon methods. The phylogenetic 14 relationship of modern and ancient populations of Adélie penguins is shown in 15 supplementary Figure S2. First, to estimate the neutral evolutionary rate we analyzed 16 only the synonymous positions (fourfold degenerate sites) of the mitochondrial 17 protein-coding regions, which are known to be largely free from selection [16]. We 18 used a Bayesian statistics-based Markov chain Monte Carlo (MCMC) method [17], as 19 well as Maximum Likelihood [18] approaches to estimate evolutionary rates. The 20 Bayesian MCMC analysis showed that the mitogenomic rate of evolution at 21 synonymous sites $\left(\mu_{\mathrm{S}}\right)$ is 0.073 (HPD, 95\% Highest Posterior Density intervals 0.025 $22-0.123$ substitutions per site per million years (s/s/My) (Figure 2). This rate estimate 23 was obtained using a general time-reversible model of evolution. To account for rate 24 variation among nucleotide positions we used a discrete gamma distribution of six 25 rate categories. The rate was then estimated assuming a constant population size and 
1 a relaxed uncorrelated log-normal molecular clock. The estimates remained almost

2 the same when modifying a range of prior assumptions. To avoid any methodological

3 bias we also estimated the rate of evolution using a Maximum Likelihood method,

4 which resulted in a rate of $0.062 \mathrm{~s} / \mathrm{s} / \mathrm{My}(\mathrm{CI} 0.03-0.09)$. This rate is slightly less than,

5 but comparable to that estimated using the Bayesian MCMC approach (Figure 2).

6 Furthermore, a similar rate was obtained using a simple distance based serial UPGMA

7 method [19] $(0.072 \mathrm{~s} / \mathrm{s} / \mathrm{My}$, CI $0.0-0.317)$.

\section{Evolutionary rates from modern penguin samples}

10 Next we estimated the rate of mitogenome evolution in Adélie penguins using only

11 the modern samples. We calculated the ratio of the mean pair-wise divergences for

12 noncoding sites at the HVR-I $\left(d_{\mathrm{H}}\right)$ and synonymous sites $\left(d_{\mathrm{S}}\right)$ using mitochondrial

13 genomes recovered from 12 modern Adélie penguins. The rate for synonymous

14 positions $\left(\mu_{\mathrm{S}}\right)$ was estimated as $\mu_{\mathrm{S}}=\mu_{\mathrm{H}} \cdot\left(d_{\mathrm{S}} / d_{\mathrm{H}}\right)$, where $\mu_{\mathrm{H}}$ is the neutral mutation rate

15 at the HVR-I region, which was previously estimated as $0.55 \mathrm{~s} / \mathrm{s} / \mathrm{My}$ [20]. Our

16 current analysis shows that the synonymous sites from modern Adélie penguin

17 mitochondrial genomes evolve at a rate $\left(\mu_{\mathrm{S}}\right)$ of $0.054(\mathrm{CI} 0.031-0.090) \mathrm{s} / \mathrm{s} / \mathrm{My}$

18 (Figure 2). This rate is not significantly different from those obtained using ancient

19 DNA methods as the confidence intervals are almost the same.

21 Inferring tempo of evolution at constrained sites in the mitochondrial

22 genome

23 Estimation of evolutionary rates at constrained sites (nonsynonymous sites and

24 RNAs) is prone to large stochastic errors owing to the low divergence in population

25 data. However the average rate of evolution at constrained sites can be estimated 
1 using the synonymous evolutionary rate. The neutral theory defines the rate of

2 evolution $(K)$ at constrained sites as $K=\mu \cdot f_{0}$ where $\mu$ is the mutation rate and $f_{0}$ is the

3 fraction of neutral positions (or neutral mutations) [21]. Since the rate of evolution at

4 synonymous sites $\left(\mu_{S}\right)$ of mitochondrial genes is known to be similar to the mutation

5 rate $(\mu)$ and the divergence at these sites $\left(d_{\mathrm{S}}\right)$ largely reflects the accumulation of

6 neutral mutations over time [16], the evolutionary rates in the constrained sites can be

7 estimated as $K_{\mathrm{X}}=\mu_{S} \cdot\left(d_{\mathrm{X}} / d_{\mathrm{S}}\right)$ where $K_{\mathrm{X}}$ and $d_{\mathrm{X}}$ are the rate of evolution and the

8 divergence in constrained sites respectively. The ratio $\left(d_{\mathrm{X}} / d_{\mathrm{S}}\right)$ is actually the fraction

9 of neutrally evolving positions $\left(f_{0}\right)$. Using this approach, we estimated that the

10 nonsynonymous rate of change for the mitochondrial genome of Adélie penguins is

$11 \quad 0.005-0.007 \mathrm{~s} / \mathrm{s} / \mathrm{My}$.

12 The synonymous molecular rate $(0.054-0.073 \mathrm{~s} / \mathrm{s} / \mathrm{My})$ differs from the 13 nonsynonymous rate by more than 10 times (Figure 2). The mitochondrial tRNAs $14(0.015-0.020 \mathrm{~s} / \mathrm{s} / \mathrm{Mya})$ evolve almost twice as fast as rRNAs $(0.008-0.010 \mathrm{~s} / \mathrm{s} / \mathrm{My})$.

15 The rate of evolution in the complete mitochondrial molecule (excluding the control 16 region) is $0.018-0.024 \mathrm{~s} / \mathrm{s} / \mathrm{My}$, which is $1.8-2.4$ times faster than the classical 17 phylogenetic rate of $0.01 \mathrm{~s} / \mathrm{s} / \mathrm{My}$ that has been used for several decades $[1,22]$. A 18 previous study on the mitochondrial genomes of the extinct mammoth also suggests 19 that the rate based on internal calibrations (within mammoths) is $\sim 1.6$ times higher 20 than that obtained using external (mammoth-elephant) calibration [23].

21 By contrast, the synonymous substitution rate $(0.054-0.073 \mathrm{~s} / \mathrm{s} / \mathrm{My})$ estimated 22 here is five to seven times higher than previous phylogenetic rate estimates [1-4] and 23 significantly higher than those based on intra-specific comparisons within human $24(0.048-0.052 \mathrm{~s} / \mathrm{s} / \mathrm{My})$ [14] and Neanderthal (0.036-0.042 s/s/My) [24] populations. 25 These results clearly argue against the use of the classical $1 \%$ rate per lineage (or the 
$12 \%$ rule as it is commonly known) to study the evolution or genetics of the individual

2 species.

4 Time dependency of molecular rates

5 Previously it has been suggested that rates of molecular evolution are time dependent

6 and that rates estimated over short timescales are higher than long-term evolutionary

7 rates $[6,9]$. To detect any time dependency, we first determined the coalescence

8 times of shallow and deep branches of modern Adélie penguin populations.

9 Previously studies have shown the presence of two distinct lineages of Adélie 10 penguins namely Antarctic (A) and Ross Sea (RS) lineages [7, 15]. Using the 11 complete mitogenomic evolutionary rate, we estimated the coalescence time between 12 the lineages $(\mathrm{A} / \mathrm{RS})$ to be 62,000 years $(62 \mathrm{Ky})$ and the coalescence time within 13 individual lineages are $18 \mathrm{Ky}$ and $19 \mathrm{Ky}$ for $\mathrm{A}$ and $\mathrm{RS}$ respectively. We then 14 estimated the root heights of the most recent common ancestor ( $\mu t_{\mathrm{MRCA}}$, substitutions 15 per site) of A, RS and A/RS lineages using synonymous (third codon) positions and 16 constrained (nonsynoymous sites+tRNAs+rRNAs) positions. The $\mu t_{\mathrm{MRCA}} \mathrm{s}$ were 17 estimated using the program MCMCcoal [25] and using the coalescence times, the 18 rate of evolution at constrained- and at synonymous sites for inter- and intra-lineages 19 of Adélie penguins were computed.

20 Our examination of the UPGMA trees (Supplementary Figure S3) reveals that the 21 node-heights of A and RS lineages are much longer (with respect to the inter-lineage $22-\mathrm{A} / \mathrm{RS}$ height) for constrained sites (Figure $\mathrm{S} 3 \mathrm{~b}$ ) than those constructed using 23 synonymous sites (Figure S3a). The rate of evolution at constrained sites is more than 24 two fold higher within A (0.02 s/s/My, HPD 0.014-0.033 s/s/My) and within RS $25(0.017 \mathrm{~s} / \mathrm{s} / \mathrm{My}, \mathrm{HPD} 0.011-0.034 \mathrm{~s} / \mathrm{s} / \mathrm{My})$ lineages, compared to that obtained for 
1 between A and RS lineages (0.008 s/s/My, HPD 0.007-0.01 s/s/My) (Figure 3).

2 However the evolutionary rates at synonymous sites estimated for intra- and inter-

3 lineages are very similar (Figure 3 ). In a recent study a comparable pattern was

4 observed in human populations [14]. The rate of evolution at third codon positions

5 was found to be similar to that recorded within human populations $(0.052 \mathrm{~s} / \mathrm{s} / \mathrm{My})$ and

6 between humans and chimpanzees $(0.048 \mathrm{~s} / \mathrm{s} / \mathrm{My})$. By contrast, the nonsynonymous

7 rate of evolution within human populations $(0.013 \mathrm{~s} / \mathrm{s} / \mathrm{My})$ was 3.4 times higher than

8 that estimated between humans and chimpanzees $(0.0038 \mathrm{~s} / \mathrm{s} / \mathrm{My})$. The rate

9 difference (3.4 times) recorded for the hominid data is higher than that reported in this

10 study (2 times, Figure 3 ). This is because the hominid comparison is on a much

11 deeper time scale (between species) than our comparison involving two lineages of

12 the same species. Since multiple substitutions are not an issue for comparisons within

13 a species or for closely related species (particularly for synonymous sites), there is no

14 reason to expect any significant variation in the rate of neutral evolution at different

15 timescales. These results suggest that the time dependency of evolutionary rates

16 holds true only for the sites under selection and not for the neutral synonymous sites.

17 Furthermore we examined the effect of time dependency on the rate for the 18 mitochondrial hyper-variable region (HVR), which is also known to evolve under 19 neutrality. The rate of evolution between lineages (A/RS) (0.496 s/s/My, HPD 0.399$20 \quad 0.615 \mathrm{~s} / \mathrm{s} / \mathrm{My})$ was not significantly different from that within the Antarctic lineage 21 (A) (0.413 s/s/My, HPD 0.288-0.675 s/s/My), but the rate within Ross Sea lineage (RS) (0.798 s/s/My, HPD 0.511-1.59 s/s/My) was higher than the inter-lineage rate.

23 Since the rate of evolution within the RS lineage is based on only three sequences (see 24 Supplementary Figure S3) as opposed to nine sequences for the A lineage, the higher 25 rate for the RS lineage could be simply due to stochastic errors. Furthermore the 
1 well-known mutational hot spots in HVR could also influence the rate estimation.

2 For instance recently Howell et al. [26] have shown that, while the rate of evolution

3 of a large fraction of the HVR region is comparable to that for synonymous sites,

4 some other sites mutate an order of magnitude faster than the remainder. Therefore

5 the rate difference observed within A and within RS lineages is most likely a result of

6 mutational processes and not due to purifying selection or the effects of time 7 dependency.

\section{Concluding remarks}

10 For decades, phylogenetic estimates of rates of molecular change have been used 11 widely in evolutionary studies but the field has recently been characterized by debates 12 about the use of intra-specific rate estimates [6, 7]. Previous studies of the 13 mitochondrial hyper-variable regions (HVR) suggested much higher intra-specific 14 evolutionary rates in comparison to classical phylogenetic rates estimated using 15 multiple species. However HVR rates cannot be used to infer molecular rates for 16 mitochondrial genomes as this region evolves much faster than rest of the genome $17[12,27]$. By contrast, the evolutionary rate for synonymous positions reported here is 18 useful to infer the rates of constrained protein coding sites, tRNA and rRNAs, which 19 will help to understand the patterns of purifying selection acting at the population 20 level. Furthermore we have shown that the high neutral rate reported in this study is 21 similar between shallow and deep branches. Therefore this time independent rate can 22 be used to study the recent evolutionary histories of many avian species.

We thank Griffith University, the Allan Wilson Centre for Molecular Ecology and Evolution and the 
1 of the Antarctica New Zealand (DML and CDM), the Italian Antarctic Programme (CB) and the

2 National Science Foundation and National Geographic Society (SE). The radiocarbon ages were

3 provided by the Geochron Laboratory - Krueger Enterprise Inc., Cambridge, Massachusetts

4 (conventional and AMS, GX-), the IsoTrace Radiocarbon Laboratory, Toronto (AMS, TO-),

5 NOSAMS, Woods Hole Oceanographic Institute (AMS, OS-) and the New Zealand Institute of

6 Geological and Nuclear Sciences, Lower Hutt, New Zealand (AMS, NZA-). We are grateful to

7 reviewers of this paper for their constructive and helpful comments.

8

9

10 
1 References

2

3 1. Shields, G.F., and Wilson, A.C. (1987) Calibration of mitochondrial DNA

4 evolution in geese. J Mol Evol 24, 212-217

5 2. Lovette, I.J. (2004) Mitochondrial dating and mixed support for the "2\% rule"

6 in birds. Auk $121,1-6$

7 3. Pereira, S.L., and Baker, A.J. (2006) A mitogenomic timescale for birds

8 detects variable phylogenetic rates of molecular evolution and refutes the standard

9 molecular clock. Mol Biol Evol 23, 1731-1740

10 4. Packert, M., et al. (2007) Calibration of a molecular clock in tits (Paridae) -

11 Do nucleotide substitution rates of mitochondrial genes deviate from the $2 \%$ rule?

12 Molecular Phylogenetics and Evolution 44, 1-14

13 5. Marko, P.B. (2002) Fossil calibration of molecular clocks and the divergence

14 times of geminate species pairs separated by the Isthmus of Panama. Molecular

15 Biology and Evolution 19, 2005-2021

16 6. Ho, S.Y., and Larson, G. (2006) Molecular clocks: when times are a-changin'.

17 Trends Genet 22, 79-83

18 7. Lambert, D.M., et al. (2002) Rates of evolution in ancient DNA from Adélie 19 penguins. Science $295,2270-2273$

20 8. Ho, S.Y.W., et al. (2005) Time dependency of molecular rate estimates and

21 systematic overestimation of recent divergence times. Molecular Biology and 22 Evolution 22, 1561-1568

23 9. Penny, D. (2005) Evolutionary biology - Relativity for molecular clocks. 24 Nature 436, 183-184 
1 10. Heyer, E., et al. (2001) Phylogenetic and familial estimates of mitochondrial

2 substitution rates: Study of control region mutations in deep-rooting pedigrees.

3 American Journal of Human Genetics 69, 1113-1126

4 11. Nabholz, B., et al. (2008) Strong variations of mitochondrial mutation rate

5 across mammals - the longevity hypothesis. Molecular Biology and Evolution 25,

$6 \quad 120-130$

7 12. Soares, P., et al. (2009) Correcting for Purifying Selection: An Improved

8 Human Mitochondrial Molecular Clock. American Journal of Human Genetics 84,

$9 \quad 740-759$

10 13. Kivisild, T., et al. (2006) The role of selection in the evolution of human 11 mitochondrial genomes. Genetics $172,373-387$

12 14. Endicott, P., and Ho, S.Y. (2008) A Bayesian evaluation of human 13 mitochondrial substitution rates. Am J Hum Genet 82, 895-902

14 15. Ritchie, P.A., et al. (2004) Ancient DNA enables timing of the Pleistocene 15 origin and Holocene expansion of two Adélie penguin lineages in Antarctica. 16 Molecular Biology and Evolution 21, 240-248

17 16. Yang, Z., and Nielsen, R. (2008) Mutation-selection models of codon 18 substitution and their use to estimate selective strengths on codon usage. Mol Biol 19 Evol 25, 568-579

20 17. Drummond, A.J., and Rambaut, A. (2007) BEAST: Bayesian evolutionary 21 analysis by sampling trees. BMC Evol Biol 7, 214

22 18. Rambaut, A. (2000) Estimating the rate of molecular evolution: incorporating 23 non-contemporaneous sequences into maximum likelihood phylogenies. 24 Bioinformatics 16, 395-399 
1 19. Drummond, A.J., et al. (2002) Estimating mutation parameters, population

2 history and genealogy simultaneously from temporally spaced sequence data.

3 Genetics 161, 1307-1320

4 20. Millar, C.D., et al. (2008) Mutation and evolutionary rates in Adélie penguins

5 from the Antarctic. PLoS Genet 4, e1000209

6 21. Kimura, M. (1983) The Neutral Theory of Molecular Evolution. Cambridge

$7 \quad$ University press

8 22. Garcia-Moreno, J. (2004) Is there a universal mtDNA clock for birds? Journal

9 of Avian Biology 35, 465-468

10 23. Gilbert, M.T.P., et al. (2008) Intraspecific phylogenetic analysis of Siberian

11 woolly mammoths using complete mitochondrial genomes. Proceedings of the

12 National Academy of Sciences of the United States of America 105, 8327-8332

13 24. Briggs, A.W., et al. (2009) Targeted Retrieval and Analysis of Five

14 Neandertal mtDNA Genomes. Science 325, 318-321

15 25. Rannala, B., and Yang, Z.H. (2003) Bayes estimation of species divergence 16 times and ancestral population sizes using DNA sequences from multiple loci. 17 Genetics $164,1645-1656$

18 26. Howell, N., et al. (2007) Relative rates of evolution in the coding and control 19 regions of African mtDNAs. Molecular Biology and Evolution 24, 2213-2221

20 27. Quinn, T.W. (1992) The genetic legacy of Mother Goose-phylogeographic 21 patterns of lesser snow goose Chen caerulescens caerulescens maternal lineages. 22 Molecular Ecology 1, 105-117 


\section{$1 \quad$ List of Figure Legends}

2

Figure 1. The location of ancient and modern samples of Adélie penguin used in this study. (a) Ross Sea Coast colonies and (b) Ross and Beaufort Island colonies sampled. Red indicates modern and black indicates ancient samples.

Figure 2. Mitogenomic rates of evolution in Adélie penguins.

Using synonymous positions (fourfold degenerate sites) of the mitochondrial genomes of eight ancient and 12 modern Adélie penguins, the evolutionary rate at synonymous sites $\left(\mu_{\mathrm{S}}\right)$ was estimated by Bayesian statistic based MCMC (red column) and a Maximum likelihood approach (yellow column). In addition, using only the modern genomes the synonymous rate was also inferred using the ratio of the average pair-wise divergence in the synonymous positions $\left(d_{S}\right)$ to that in the hyper-variable region $\left(d_{H}\right)$ and using the mutation rate $\left(\mu_{H}\right)$ of the latter, as $\mu_{\mathrm{S}}=\mu_{\mathrm{H}}\left(d_{\mathrm{S}} / d_{\mathrm{H}}\right)$ where $\mu_{\mathrm{H}}=0.55(0.32-0.92)$ substitutions/site/million years as reported from a previous study [20] (blue column). The evolutionary rates in different mitochondrial regions $\left(K_{\mathrm{X}}\right)$ are estimated as $K_{\mathrm{X}}=\mu_{\mathrm{S}}\left(d_{\mathrm{X}} / d_{\mathrm{S}}\right)$ where $d_{\mathrm{X}}$ is the average pair-wise distance obtained for the given region $X$ (e.g. tRNA). The error bars show $95 \%$ HPD intervals.

Figure 3. Rate of evolution for within and between the two lineages of modern Adelie penguins.

Evolutionary rates were estimated using synonymous $\left(3^{\text {rd }}\right.$ codon) positions (a) and constrained positions (nonsynonymous sites + tRNAs + rRNAs) (b). The coalescence times for within and between lineages were estimated by the program BEAST [17] using the rate obtained for the complete mitochondrial genome. The evolutionary rates for $A$ and $R$ lineages and that between them (AR) were obtained using the respective coalescence times and the root heights of most recent common ancestor $\left(\mu t_{\mathrm{MRCA}}\right)$ estimated by MCMCcoal [25] using synonymous and constrained sites. The error bars are based on the 95\% HPD intervals obtained from coalescence times estimation. 


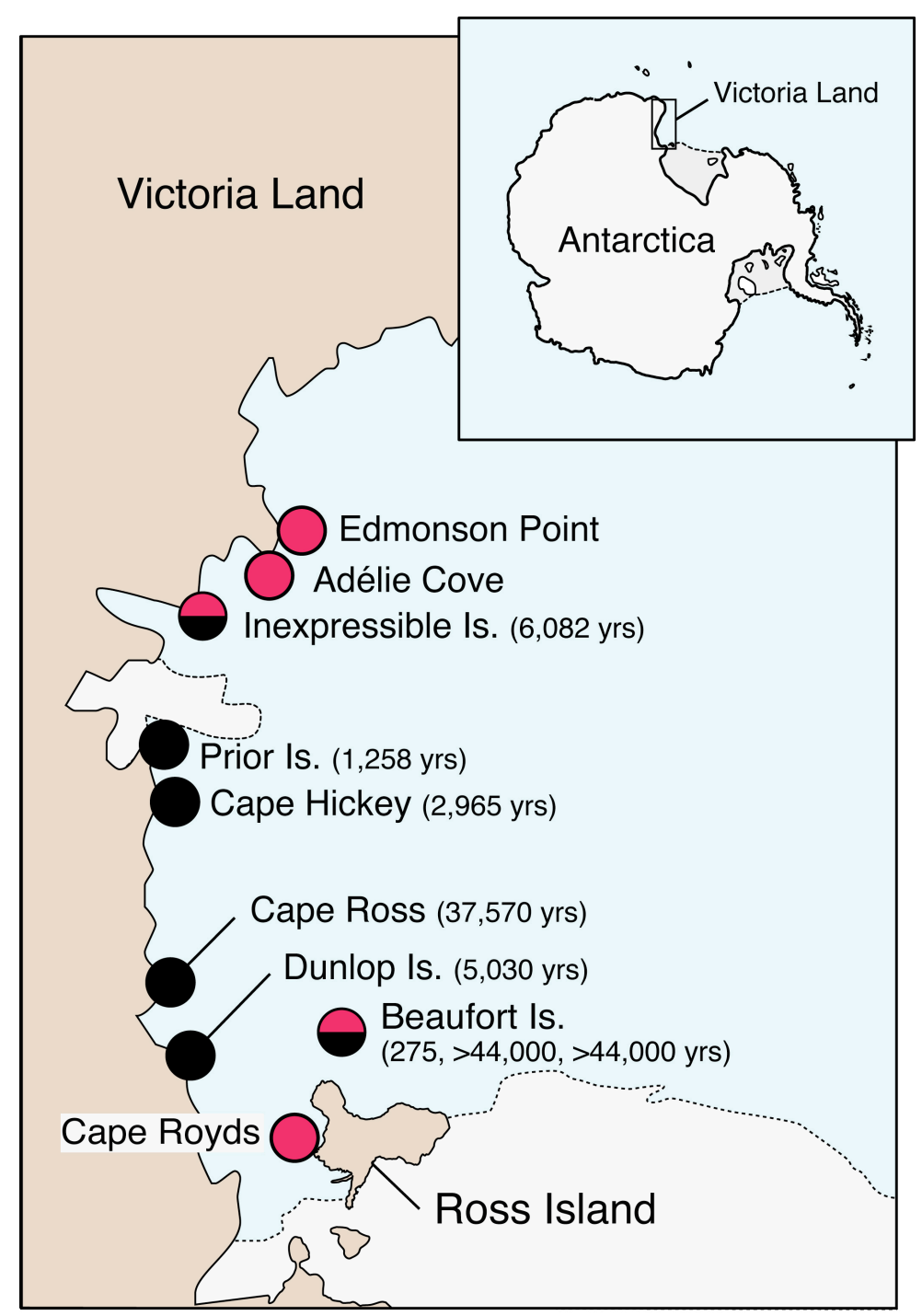

Figure 1. Subramanian et al. 2009

1 


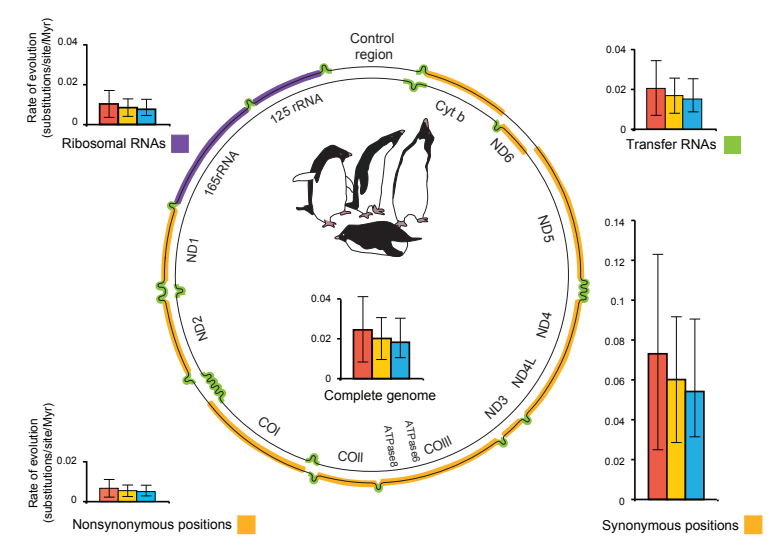

Figure 2. Subramanian et al. 2009 


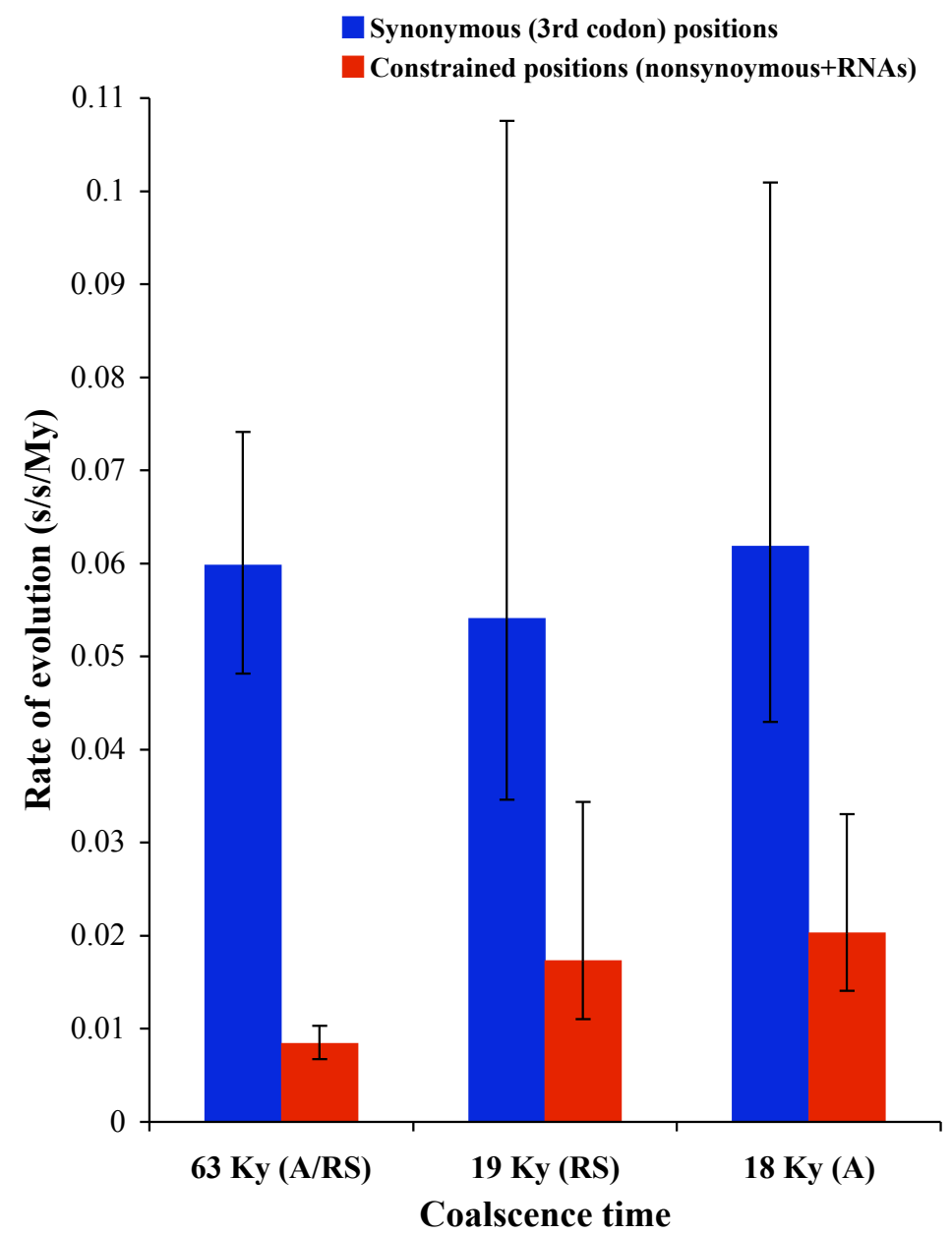

Figure 3. Subramanian et al. 2009 
3 High mitogenome-wide rates of evolution and time

4 dependency

5 Sankar Subramanian ${ }^{1,2}$, Dee R. Denver ${ }^{3,4}$, Craig D. Millar ${ }^{2}$, Tim Heupink ${ }^{3}$, Angelique

6 Aschrafi $^{3,5}$, Steven D. Emslie ${ }^{6}$ Carlo Baroni ${ }^{7}$ and David M. Lambert ${ }^{1,3 *}$

${ }^{1}$ Griffith School of Environment and the School of Biomolecular and Physical Sciences, Griffith University, 170 Kessels Road, Nathan, QId 4111 Australia.

${ }^{2}$ Allan Wilson Centre for Molecular Ecology and Evolution, School of Biological Sciences, University of Auckland, Private Bag 92019, Auckland, New Zealand.

${ }^{3}$ Allan Wilson Centre for Molecular Ecology and Evolution, Institute of Molecular BioSciences, Massey University, Auckland, New Zealand.

${ }^{4}$ Department of Zoology and Center for Genome Research and Biocomputing, Oregon State University, Corvallis, Oregon, USA.

${ }^{5}$ Department of Cell Biology, The Scripps Research Institute, La Jolla, California 92037, USA.

${ }^{6}$ Department of Biology and Marine Biology, University of North Carolina, Willmington, North Carolina, 18 USA.

${ }^{7}$ Dipartmento Scienze della Terra, Universita` di Pisa, and Consiglio Nazionale Ricerche, Centro Studio Geologia Strutturale, Pisa, Italy.

\section{DNA extraction}

Shavings of bone were taken from samples using a sterile surgical blade. About $200 \mathrm{mg}$ of bone material was transferred to a sterile Eppendorf tube. In between samples bench area and blade were cleaned using $10 \%$ bleach. After adding $1.5 \mathrm{ml}$ of $0.5 \mathrm{M}$ EDTA and $0.75 \mathrm{mg}$ of proteinase-K, the samples were rotated overnight at $56^{\circ} \mathrm{C}$. Samples were then extracted once with Tris-saturated phenol, once with Trissaturated phenol: chloroform: isoamyl-alcohol (25:24:1) and finally once with chloroform: isoamyl-alcohol (24:1). This extracted solution was then concentrated to $50 \mu \mathrm{L}$ on a Vivaspin-30 (Viva Science, U.K.) membrane. The sample was then purified using QIAGEN DNA mini kit eluting in $50 \mu \mathrm{L} 1 \times \mathrm{TE}$ and stored at $-20^{\circ} \mathrm{C}$. All DNA extractions and setting up of PCR's from sub-fossil material were conducted in a dedicated ancient DNA laboratory, which underwent regular decontamination with

Corresponding author: Lambert, D.M. (d.lambert@griffith.edu.au). 
UV-irradiation and hypochloride treatment. Mock DNA extractions and blank PCR's were continually screened for contamination.

Multiplex PCR and Sequencing Modern samples were extracted using a 10\% Chelex 100 (Sigma-Aldrich) solution in $100 \mathrm{mM}$ Tris pH8.8. Amplification of the mitochondrial genomes was performed using a high throughput plate set up. The set up of the multiplex PCR is as described in a previously published protocol (Römlper et al. 2006). All samples where amplified using a two stage multiplex PCR, using 51 overlapping primer pairs and another 51 nested primer pairs (Table S1). The first stage $50 \mu 1$ multiplex reaction contained final concentrations of: $0.4 \mu \mathrm{M}$ of each primer (two reactions with 25 or 26 pairs), 1x PCR Buffer, 2.5mM $\mathrm{MgCl}_{2}, 0.2 \mathrm{mM}$ dNTPs, $1 \mathrm{mg} / \mathrm{ml}$ BSA, 1.25 units of Platinum Taq DNA polymerase (Invitrogen) and $1 \mu 1$ of DNA extract. The thermo cycling was performed as follows: $4 \min 95^{\circ} \mathrm{C}, 35 \mathrm{x}\left(30 \mathrm{sec} 95^{\circ} \mathrm{C}, 30 \mathrm{sec} 55^{\circ} \mathrm{C}, 45 \mathrm{sec}\right.$ $72^{\circ} \mathrm{C}$ ), $10 \mathrm{~min} 72^{\circ} \mathrm{C}$. The second stage $50 \mu 1$ simplex reaction contained final concentrations of: $0.25 \mu \mathrm{M}$ of each primer (51 reactions with one pair), 1x PCR Buffer, $2.5 \mathrm{mM} \mathrm{MgCl}_{2}, 0.2 \mathrm{mM}$ dNTPs, $1 \mathrm{mg} / \mathrm{ml} \mathrm{BSA}, 1.25$ units of Platinum Taq DNA polymerase and $1 \mu 1$ of 1:50 diluted $1^{\text {st }}$ stage PCR product. The thermo cycling was performed as described for the first stage reaction.

PCR reactions were purified for sequencing using the AMPure kit according to manufacturer's protocol. Samples where then subjected to ethanol precipitation and sequenced using the BigDye Terminator v3.1 cycle sequencing kit (ABI), according to manufacturer's protocol.

\section{Evolutionary rate estimation}

The length of Adelie penguin mitochondrial genomes recovered varies between 12.5 $16.1 \mathrm{~kb}$ (Figure S1). Amino acid sequences of each protein coding gene and the nucleotide sequences of each tRNA and rRNA gene were aligned by CLUSTAL-W [2]. The cDNA alignments of protein coding genes were created using the alignments of amino acid sequences. We employed a complete site deletion method by excluding the nucleotide positions that contained an alignment gap in one or more sequences. Fourfold and zerofold degenerate sites were extracted from the cDNA alignments. Our final dataset consists of 1349 fourfold degenerate positions, 4860 zerofold degenerate positions, 1136 tRNA and 1089 rRNA sites from 12 modern and eight ancient Adélie penguins. Pair-wise distance estimations were performed using MEGA [3].

To estimate the neutral evolutionary rate, only the fourfold degenerate sites were used. First a simple distance based evolutionary rate was estimated using a serial UPGMA method employed in the software Pebble under a HKY+Gamma model of evolution [4]. Furthermore Pebble was used to construct an UPGMA tree of modern and ancient Adélie penguins by accounting for the ages of the ancient sequences (Figure S2). The program TipDate [5] implemented in PAML [6] was used to estimate the rate of evolution by the maximum likelihood method using a GTR+Gamma model of evolution and the UPGMA tree was used to provide the topology.

We also employed Bayesian statistics based on a Markov chain Monte Carlo approach using the software BEAST [7] assuming a relaxed molecular clock, constant population size and GTR+Gamma model of evolution. Wide uniform prior 
1 distributions were used to avoid any bias due to restricted prior assumptions. The rate 2 estimate and the highest posterior density intervals were similar when varying a 3 number of a priori assumptions. The rate estimates were sampled once every 1000 4 cycles from the total MCMC chain length of 10,000,000 after discarding the initial 5 1,000,000 burn-in 1,000,000 steps. The posterior distributions were examined using 6 the software TRACER [8] and the Effective Sample Sizes (ESS) for mean 7 evolutionary rate, population size and posterior likelihood were found to be $>100$ for 8 all the models used.

10 To estimate the root height ( $\mu t_{\mathrm{MRCA}}$, substitutions per site) of the most recent common 11 ancestor the MCMCcoal was used [9]. $\mu t_{\mathrm{MRCA}} \mathrm{s}$ were A, RS and A/RS lineages using 12 synonymous (third) positions and also using constrained sites including

Table S1. External and Internal primers used to amplify complete Adélie penguin mitochondrial genomes. Plate $=$ name of master primer plate. Row and Col depict location in master primer plates. Gapdh and Zenk are nuclear loci used to monitor the presence of nuclear DNA.

\begin{tabular}{|c|c|c|c|c|}
\hline PLATE & ROW & COL & PRIMERNAME & PRIMER SEQ \\
\hline Pa_mt_External & A & 1 & PaMTOF01 & AACCAAAGCATGGCACTGAAGAT \\
\hline Pa_mt_External & $E$ & 1 & PaMTOF02 & TTAACTGTCACACGGCGTAAAGA \\
\hline Pa_mt_External & A & 3 & PaMTOF03 & TCGCCTGAAAGAACAACAGTGA \\
\hline Pa_mt_External & $E$ & 3 & PaMTOF04 & AACACAAAGCACTCAGCTTACACCT \\
\hline Pa_mt_External & $A$ & 5 & PaMTOF05 & AGTGGGATGACTTGTTAGTAGAGGT \\
\hline Pa_mt_External & $E$ & 5 & PaMTOF06 & AAGCGCAAGCTTACATCACCACATT \\
\hline Pa_mt_External & $A$ & 7 & PaMTOF07 & ACTGTCTCTTGCAGACAATCAATG \\
\hline Pa_mt_External & $E$ & 7 & PaMTOF08 & TTTACGACCTCGATGTTGGATCA \\
\hline Pa_mt_External & $A$ & 9 & PaMTOF09 & ATCCCAATCCTAGTCGCAGT \\
\hline Pa_mt_External & $E$ & 9 & PaMTOF10 & TATGATCAGGGTGGGCCTCAAA \\
\hline Pa_mt_External & $A$ & 11 & PaMTOF11 & GCAGGTCCATTCGCTTTATTCTT \\
\hline Pa_mt_External & $E$ & 11 & PaMTOF12 & СААСССТСТСАТTTССТGAGATACA \\
\hline Pa_mt_External & $\mathrm{B}$ & 1 & PaMTOF13 & TTCACAGGACAGTGAGACATCA \\
\hline Pa_mt_External & $\mathrm{F}$ & 1 & PaMTOF14 & СССАААСТТАСССТАТТААССТТСТАСС \\
\hline Pa_mt_External & $\mathrm{B}$ & 3 & PaMTOF15 & AGTTGCCATCCTTACCTCCTTAT \\
\hline Pa_mt_External & $\mathrm{F}$ & 3 & PaMTOF16 & AAAGGACTACAGCCTAACGCCT \\
\hline Pa_mt_External & $\mathrm{B}$ & 5 & PaMTOF17 & CCCGACATAGCATTTCCC \\
\hline Pa_mt_External & $\mathrm{F}$ & 5 & PaMTOF18 & ACCGAAACCTAAACACTACCT \\
\hline Pa_mt_External & B & 7 & PaMTOF19 & TGGGCTTCATCTTCCTCTTCACTAT \\
\hline Pa_mt_External & $\mathrm{F}$ & 7 & PaMTOF20 & ATTCATCATCTGAGAAGCCCT \\
\hline Pa_mt_External & $\mathrm{B}$ & 9 & PaMTOF21 & AATTCGGATTCCAAGATGCCT \\
\hline Pa_mt_External & $\mathrm{F}$ & 9 & PaMTOF22 & ACATAATCCCAACAACAGACC \\
\hline Pa_mt_External & B & 11 & PaMTOF23 & AATCGTAGTAGAATCTGCCC \\
\hline Pa_mt_External & $\mathrm{F}$ & 11 & PaMTOF24 & AGGAATTCCGCTAATCCTACT \\
\hline Pa_mt_External & $\mathrm{C}$ & 1 & PaMTOF25 & AATCGAAACCACTAGCCTACTCA \\
\hline Pa_mt_External & G & 1 & PaMTOF26 & TAACCCTTGGCCTACTTTCCATA \\
\hline Pa_mt_External & $\mathrm{C}$ & 3 & PaMTOF27 & AATCCACGCACTAACCCTAACAA \\
\hline Pa_mt_External & G & 3 & PaMTOF28 & ААТСАСАТТСАТАСТСАСССТСТСС \\
\hline Pa_mt_External & C & 5 & PaMTOF29 & ATGAGTGAACACAAGGAGGCCTAGA \\
\hline Pa_mt_External & G & 5 & PaMTOF30 & ATACTAGTTGCCTCAACCCGAA \\
\hline
\end{tabular}


Pa_mt_External C Pa_mt_External $G$ Pa_mt_External C Pa_mt_External $G$ Pa_mt_External C Pa_mt_External $G$ Pa_mt_External $D$ $\mathrm{Pa}$ _mt_External $\mathrm{H}$ Pa_mt_External D $\mathrm{Pa}$ mt_External $\mathrm{H}$ Pa_mt_External $D$ $\mathrm{Pa}$ mt_External $\mathrm{H}$ Pa_mt_External D $\mathrm{Pa}$ _mt_External $\mathrm{H}$ Pa_mt_External $\mathrm{D}$ $\mathrm{Pa}$ mt_External $\mathrm{H}$ Pa_mt_External D $\mathrm{Pa}$ _mt_External $\mathrm{H}$ $\mathrm{Pa}$ mt_External A $\mathrm{Pa}$ at_External $\mathrm{E}$ $\mathrm{Pa}$ at_External A Pa_mt_External $E$ Pa_mt_External A $\mathrm{Pa}$ mt_External $\mathrm{E}$ $\mathrm{Pa}$ mt_External A Pa_mt_External $E$ Pa_mt_External A Pa_mt_External $E$ Pa_mt_External A $\mathrm{Pa}$ mt_External $\mathrm{E}$ Pa_mt_External B $\mathrm{Pa}$ _mt_External $\mathrm{F}$ Pa_mt_External B $P a \_m t$ External $\mathrm{F}$ Pa_mt_External B $\mathrm{Pa}$ _mt_External $\mathrm{F}$ Pa_mt_External B $\mathrm{Pa}$ _mt_External $\mathrm{F}$ $\mathrm{Pa}$ at_External $\mathrm{B}$ $\mathrm{Pa}$ mt_External $\mathrm{F}$ $\mathrm{Pa}$ mt_External $\mathrm{B}$ $\mathrm{Pa}$ at_External $\mathrm{F}$ $\mathrm{Pa}$ at_External C Pa_mt_External $G$ Pa_mt_External C Pa_mt_External $G$ Pa_mt_External C Pa_mt_External $G$ Pa_mt_External C Pa_mt_External $G$ Pa_mt_External C Pa_mt_External $G$
PaMTOF32

PaMTOF33

PaMTOF34

PaMTOF35

PaMTOF36

PaMTOF37

PaMTOF38

PaMTOF39

PaMTOF40

PaMTOF41

PaMTOF42

PaMTOF43

PaMTOF44

PaMTOF45

PaMTOF46

PaMTOF47

PaMTOF48

PaMTOR01

PaMTOR02

PaMTOR03

PaMTOR04

PaMTOR05

PaMTOR06

PaMTOR07

PaMTOR08

PaMTOR09

PaMTOR10

PaMTOR11

PaMTOR12

PaMTOR13

PaMTOR14

PaMTOR15

PaMTOR16

PaMTOR17

PaMTOR18

PaMTOR19

PaMTOR20

PaMTOR21

PaMTOR22

PaMTOR23

PaMTOR24

PaMTOR25

PaMTOR26

PaMTOR27

PaMTOR28

PaMTOR29

PaMTOR30

PaMTOR31

PaMTOR32

PaMTOR33

PaMTOR34
AAACCACCTTCAACACGAACCT AGCCCTACTAACAGCATTCATAGT TCCGGTGCAATGATCCTCATAAT ATACTACTAACAACCCAACGAGG AACCAACAAGAGCTGCTAACT GCATTACATCCTACTGAGAGTGA TACAACCGAATCGGAGACATT TAATAACCCAACTGCCCTCA AGACCTCATTATCGAAAGCCT ACCTAAACTTCTCСТССТССТTA TGCCTGACTACACAAATCCTGA ATATATTGGCCAAACCCTCGT AACAAACTGGGAGGTGTCCTA TGGTCTTGTAAGCCAAAGAGT AACCCAACAACAAGCACCAAGAT AAGTCATAGCAATTCCCGCTT CATTTAATGTACGTACTAGGAC GGACTGTCATGTAACAAACGACT ACATCTTGGGCTTATGACAGCTT AAATGTAGCCCATTTCTTCCACC TTGGGCTAGAGTTTGGCTTCAA TTCATTTCACAGGCAACCAGCTA TGGGCTAACAGGGCAAGTTTAAT TTTAAGTTCCACAGGGTCTTCTCGT AATCGTTGAACAAACGAGCCCTT TTTCGGGCTTGTATGTAGCTT AAATAGTTTGTGCTACTGCCCGT TGGGTTGAGAAACAGAATGGT TTGATTTCTTCTGCGTAGGTTCG ATTGCAATTGCTGCTGTCAGT TCTTGGTTGTGTTGAGTGTGAGA TCCTAAGTTTCTAGGTGGTTGCT TGCCGATATCTTTGTGGTTGGTT TTCTACTGTGGATGAGGCTAGT AGAATCAGAAGAGGTGTTGGT ATGTCCAATGAGGAGTTTGCT TCATTCAATGTTGGTGGCAGT TGCAGATTGCTAGGGCAACTATT TAACAACACGGTGGTCTACTT AGGCTAGTGCTGTTTCATAGCTT ACGGTTGGTAATTCATCGGTT TTAGTTGAATGAGTAGGTGGCCT TGGCCTTGAAATGTGCTTTCT AGCAATTGAGAATGGTGCTTCGT TGGCTTGTGCCAATCAGAAGTTT ATGGTCTGTTGAGCCGAAATCAA TGGTGGGAAGGAGTATGATTGTT TGAGGAAGGGTTGGATTGTGATT AGATCCTGCAATTGGTGCTTCTA AAGAATTCGGGTGTGTGTTCGTT AGGTGTTCTCGTGTGTTTGAGTT ACTTGGATTTGCACCAAGATG 
Pa_mt_External C $12 \quad$ PaMTOR35 Pa_mt_External G 12 PaMTOR36 Pa_mt_External D 2 PaMTOR37 Pa_mt_External $\mathrm{H} \quad 2 \quad$ PaMTOR38 Pa_mt_External D 4 PaMTOR39 Pa_mt_External $\mathrm{H} \quad 4 \quad$ PaMTOR40 Pa_mt_External D 6 PaMTOR41 Pa_mt_External $\mathrm{H} \quad 6 \quad$ PaMTOR42 Pa_mt_External $\mathrm{D} \quad 8 \quad$ PaMTOR43 Pa_mt_External $\mathrm{H} \quad 8 \quad$ PaMTOR44 Pa_mt_External D 10 PaMTOR45 Pa_mt_External $\mathrm{H} \quad 10 \quad$ PaMTOR46 Pa_mt_External D 12 PaMTOR47 1
TCATGTTACGAACAGTGCGAT AGGTGTTTATTGTTGAGGCGA ACATGTAGCGGCAAATAGTGT ATAGGTTGCGGTGAATGATGT TTTGTCCGCTGTTTAGTAGGT ATTGTACATCTCGGCATGTGT AGGAAGTGTAGTGCGAAGAAT TTGTGCGCTGTTTAGACTTGT AAATGCCAGCTTTGGGAGTTGGATA TCTTTGGCAGCGGATTTGTTT TTCCTGTGGTTGAAGTAACAACG TACTCGTTTAATCCGAAGGGA GTGTTCAAGCTCTGCCGTACC AAAGGGAACCAACAGTGCCAAA 


\begin{tabular}{|c|c|c|c|c|}
\hline a_mt_Internal & A & 1 & PaMTIF01 & ACTGAAGATGCCAAGATGATGCT \\
\hline $\mathrm{Pa} \_\mathrm{mt}$ Internal & A & 2 & PaMTIR01 & CATCTTGGGCTTATGACAGCTTAG \\
\hline Pa_mt_Internal & $A$ & 3 & PaMTIF03 & CCTGAAAGAACAACAGTGAGCAT \\
\hline Pa_mt_Internal & A & 4 & PaMTIR03 & TAGAGTTTGGCTTCAAGGCGAT \\
\hline Pa_mt_Internal & $A$ & 5 & PaMTIF05 & TGGGATGACTTGTTAGTAGAGGTGA \\
\hline $\mathrm{Pa}$-mt_Internal & $A$ & 6 & PaMTIR05 & GCAAGTTTAATATTTGGCTTGTTTGGG \\
\hline $\mathrm{Pa} \mathrm{mt}$ Internal & A & 7 & PaMTIF07 & CAATGAAATTGATCTTCCTGTGC \\
\hline $\mathrm{Pa}$-mt_Internal & $A$ & 8 & PaMTIR07 & ATCGTTGAACAAACGAGCCCTTA \\
\hline Pa_mt_Internal & $A$ & 9 & PaMTIF09 & ATCCTAGTCGCAGTCGCCTACC \\
\hline $\mathrm{Pa} \_\mathrm{mt}$ - Internal & $A$ & 10 & PaMTIR09 & TTTGTGCTACTGCCCGTAGTG \\
\hline Pa_mt_Internal & $A$ & 11 & PaMTIF11 & GGTCCATTCGCTTTATTCTTCTTAGC \\
\hline Pa_mt_Internal & A & 12 & PaMTIR11 & TTGATTTCTTCTGCGTAGGTTCGAC \\
\hline Pa_mt_Internal & $\mathrm{B}$ & 1 & PaMTIF13 & ACAGTGAGACATCACCCAACTAA \\
\hline $\mathrm{Pa}$-me- Internal & $\mathrm{B}$ & 2 & PaMTIR13 & GGTTGTGTTGAGTGTGAGAAATACTG \\
\hline $\mathrm{Pa} \mathrm{mt}$ Internal & B & 3 & PaMTIF15 & TTGCСАТССТTАССТССТTАТСА \\
\hline $\mathrm{Pa} \_\mathrm{mt}$ - Internal & $\mathrm{B}$ & 4 & PaMTIR15 & GCCGATATCTTTGTGGTTGGTTGAGAAT \\
\hline Pa_mt_Internal & $\mathrm{B}$ & 5 & PaMTIF17 & CCCGTATAAATAACATAAGC \\
\hline Pa_mt_Internal & $\mathrm{B}$ & 6 & PaMTIR17 & AGAAGAGGTGTTGGTATAGG \\
\hline $\mathrm{Pa}$ - mt_Internal & $\mathrm{B}$ & 7 & PaMTIF19 & TCATCTTCСТСТTCACTATCG \\
\hline Pa_mt_Internal & $\mathrm{B}$ & 8 & PaMTIR19 & TCAATGTTGGTGGCAGTTAGT \\
\hline Pa_mt_Internal & $\mathrm{B}$ & 9 & PaMTIF21 & ATTCCAAGATGCСTCATСTCCCATT \\
\hline $\mathrm{Pa} \_\mathrm{mt}$ - Internal & $\mathrm{B}$ & 10 & PaMTIR21 & TAACAACACGGTGGTCTACTTCT \\
\hline $\mathrm{Pa} \mathrm{mt}$ Internal & B & 11 & PaMTIF23 & СССАСТСАСССАСТTCGAGA \\
\hline $\mathrm{Pa}$-mt_Internal & $\mathrm{B}$ & 12 & PaMTIR23 & AGACGGTTGGTAATTCATCGGTTGT \\
\hline $\mathrm{Pa}{ }^{-} \mathrm{mt}$ - Internal & C & 1 & PaMTIF25 & TCGAAACCACTAGCCTACTCAT \\
\hline $\mathrm{Pa}$ - mt_Internal & $\mathrm{C}$ & 2 & PaMTIR25 & TGGCCTTGAAATGTGCTTTCTCGTA \\
\hline $\mathrm{Pa}{ }^{-} \mathrm{mt}$ - Internal & C & 3 & PaMTIF27 & TCCACGCACTAACCCTAACAAT \\
\hline $\mathrm{Pa} \_\mathrm{mt}$ - Internal & $\mathrm{C}$ & 4 & PaMTIR27 & GGCTTGTGCCAATCAGAAGTTTA \\
\hline Pa_mt_Internal & C & 5 & PaMTIF29 & TGAACACAAGGAGGCCTAGAGTG \\
\hline $\mathrm{Pa}$-mt_Internal & $\mathrm{C}$ & 6 & PaMTIR29 & AAGGAGTATGATTGTTGGGAGGA \\
\hline $\mathrm{Pa}{ }^{-} \mathrm{mt}$ - Internal & $\mathrm{C}$ & 7 & PaMTIF31 & TTCAACACGAACCTCCAACACGAA \\
\hline Pa_mt_Internal & C & 8 & PaMTIR31 & АTCCTGCAATTGGTGCTTCTAC \\
\hline $\mathrm{Pa}{ }^{-} \mathrm{mt}$ - Internal & C & 9 & PaMTIF33 & CGGTGCAATGATCCTCATAATCT \\
\hline $\mathrm{Pa} \_\mathrm{mt}$ - Internal & $\mathrm{C}$ & 10 & PaMTIR33 & GGTGTTCTCGTGTGTTTGAGTTT \\
\hline $\mathrm{Pa}{ }^{-} \mathrm{mt}$ - Internal & C & 11 & PaMTIF35 & AACAAGAGCTGCTAACTCCTGCAT \\
\hline $\mathrm{Pa}$-mt_Internal & C & 12 & PaMTIR35 & TCATGTTACGAACAGTGCGATG \\
\hline Pa_mt_Internal & $\mathrm{D}$ & 1 & PaMTIF37 & AACCGAATCGGAGACATTGG \\
\hline $\mathrm{Pa}$-mt_Internal & $\mathrm{D}$ & 2 & PaMTIR37 & ATGTAGCGGCAAATAGTGTGGAT \\
\hline $\mathrm{Pa}{ }^{-} \mathrm{mt}$ - Internal & $\mathrm{D}$ & 3 & PaMTIF39 & ATCGAAAGCCTAAATACCTCC \\
\hline $\mathrm{Pa} \_\mathrm{mt}$ - Internal & $\mathrm{D}$ & 4 & PaMTIR39 & TTGTCCGCTGTTTAGTAGGTT \\
\hline $\mathrm{Pa}{ }^{-} \mathrm{mt}$ - Internal & $\mathrm{D}$ & 5 & PaMTIF41 & TGACTACACAAATCCTGACC \\
\hline $\mathrm{Pa}$-mt_Internal & $\mathrm{D}$ & 6 & PaMTIR41 & TGTAGTGCGAAGAATCGGGTTA \\
\hline $\mathrm{Pa}{ }^{-} \mathrm{mt}$ - Internal & $\mathrm{D}$ & 7 & PaMTIF43 & AAACTGGGAGGTGTCCTAGCCTTA \\
\hline Pa_mt_Internal & $\mathrm{D}$ & 8 & PaMTIR43 & AGCTTTGGGAGTTGGATATGGAGGTTT \\
\hline Pa_mt_Internal & $\mathrm{D}$ & 9 & PaMTIF45 & CCCAACAACAAGCACCAAGATAA \\
\hline $\mathrm{Pa} \_\mathrm{mt}$ - Internal & $\mathrm{D}$ & 10 & PaMTIR45 & TTGAAGTAACAACGGCGGCTT \\
\hline $\mathrm{Pa}{ }^{-} \mathrm{mt}$ - Internal & $\mathrm{D}$ & 11 & PaMTIF47 & GTACTAGGACTGTTATTGTATTATCGGC \\
\hline Pa mt Internal & $\mathrm{D}$ & 12 & PaMTIR47 & TGTTCAAGCACTGCCGTACCTATTT \\
\hline $\mathrm{Pa}{ }^{-} \mathrm{mt}$ - Internal & $E$ & 1 & PaMTIF02 & TGTCACACGGCGTAAAGAGT \\
\hline Pa_mt_Internal & $E$ & 2 & PaMTIR02 & ATAGGCTATACCTTGACCTGTCTTGT \\
\hline $\mathrm{Pa}{ }^{-} \mathrm{mt}$ - Internal & $E$ & 3 & PaMTIF04 & CACTCAGCTTACACCTGAGAGATA \\
\hline Pa_mt_Internal & $E$ & 4 & PaMTIR04 & ATTTCACAGGCAACCAGCTATCA \\
\hline
\end{tabular}




\begin{tabular}{|c|c|c|c|c|}
\hline Pa_mt_Internal & $\mathrm{E}$ & 5 & PaMTIF06 & GCGCAAGCTTACATCACCACATTAT \\
\hline Pa_mt_Internal & $\mathrm{E}$ & 6 & PaMTIR06 & AGGGTCTTCTCGTCTTATGGGTTTA \\
\hline Pa_mt_Internal & $\mathrm{E}$ & 7 & PaMTIF08 & ATGTTGGATCAGGACACCCTAATG \\
\hline Pa_mt_Internal & $\mathrm{E}$ & 8 & PaMTIR08 & TTCGGGCTTGTATGTAGCTTAGGA \\
\hline Pa_mt_Internal & E & 9 & PaMTIF10 & AGGGTGGGCCTCAAACTCAAA \\
\hline Pa_mt_Internal & E & 10 & PaMTIR10 & GGGTTGAGAAACAGAATGGTAGT \\
\hline Pa_mt_Internal & $\mathrm{E}$ & 11 & PaMTIF12 & САAСССТСТСАTTTCCTGAGATACAA \\
\hline Pa_mt_Internal & $\mathrm{E}$ & 12 & PaMTIR12 & AATTGCTGCTGTCAGTAGGAGGCA \\
\hline $\mathrm{Pa}$ mt_Internal & $\mathrm{F}$ & 1 & PaMTIF14 & ССАААСТТАСССТАТТААССТТСТАССТ \\
\hline Pa_mt_Internal & $\mathrm{F}$ & 2 & PaMTIR14 & AGTTTCTAGGTGGTTGCTAGGATT \\
\hline Pa_mt_Internal & $\mathrm{F}$ & 3 & PaMTIF16 & TACAGCCTAACGCCTCGACA \\
\hline Pa_mt_Internal & $\mathrm{F}$ & 4 & PaMTIR16 & ATGAGGCTAGTAGCAGTAGGAA \\
\hline Pa_mt_Internal & $\mathrm{F}$ & 5 & PaMTIF18 & АССТАAАСАСТАССТТСТTCG \\
\hline Pa_mt_Internal & $\mathrm{F}$ & 6 & PaMTIR18 & TGTCCAATGAGGAGTTTGCTA \\
\hline Pa_mt_Internal & $\mathrm{F}$ & 7 & PaMTIF20 & TCTGAGAAGCCCTTACATCAA \\
\hline Pa_mt_Internal & $\mathrm{F}$ & 8 & PaMTIR20 & GCAGATTGCTAGGGCAACTATTA \\
\hline $\mathrm{Pa}$ mt_Internal & $\mathrm{F}$ & 9 & PaMTIF22 & ATAATCCCAACAACAGACCTTCC \\
\hline Pa_mt_Internal & $\mathrm{F}$ & 10 & PaMTIR22 & AGGCTAGTGCTGTTTCATAGCTTCT \\
\hline Pa_mt_Internal & $\mathrm{F}$ & 11 & PaMTIF24 & GAATTCCGCTAATCCTACTATCA \\
\hline Pa_mt_Internal & $\mathrm{F}$ & 12 & PaMTIR24 & TTAGTTGAATGAGTAGGTGGCCTG \\
\hline Pa_mt_Internal & G & 1 & PaMTIF26 & TTGGCCTACTTTCCATACTCCT \\
\hline Pa_mt_Internal & G & 2 & PaMTIR26 & GCAATTGAGAATGGTGCTTCGTA \\
\hline Pa_mt_Internal & G & 3 & PaMTIF28 & АСАТТСАТАСТСАСССТСТСССТ \\
\hline Pa_mt_Internal & G & 4 & PaMTIR28 & TGTTGAGCCGAAATCAACTGTCT \\
\hline Pa_mt_Internal & G & 5 & PaMTIF30 & ACTAGTTGCCTCAACCCGAACT \\
\hline Pa_mt_Internal & G & 6 & PaMTIR30 & TGAGGAAGGGTTGGATTGTGATTA \\
\hline Pa_mt_Internal & G & 7 & PaMTIF32 & СССТАCTAACAGCATTCATAGTAAA \\
\hline Pa_mt_Internal & G & 8 & PaMTIR32 & GGGTGTGTGTTCGTTCGTAATTT \\
\hline Pa_mt_Internal & G & 9 & PaMTIF34 & TACTAACAACCCAACGAGGGA \\
\hline Pa_mt_Internal & G & 10 & PaMTIR34 & TTGGATTTGCACCAAGATGG \\
\hline Pa_mt_Internal & G & 11 & PaMTIF36 & ACATCCTACTGAGAGTGAA \\
\hline Pa_mt_Internal & G & 12 & PaMTIR36 & GTGTTTATTGTTGAGGCGAGT \\
\hline Pa_mt_Internal & $\mathrm{H}$ & 1 & PaMTIF38 & ATAACCСАACTGСССТСАСССТАТ \\
\hline Pa_mt_Internal & $\mathrm{H}$ & 2 & PaMTIR38 & TTGCGGTGAATGATGTGGCTA \\
\hline Pa_mt_Internal & $\mathrm{H}$ & 3 & PaMTIF40 & TCСTCСTCСTTAGGATACTTCAA \\
\hline Pa_mt_Internal & $\mathrm{H}$ & 4 & PaMTIR40 & ATTGTACATCTCGGCATGTGTG \\
\hline Pa_mt_Internal & $\mathrm{H}$ & 5 & PaMTIF42 & ATATTGGCCAAACCCTCGTAGAA \\
\hline Pa_mt_Internal & $\mathrm{H}$ & 6 & PaMTIR42 & TTGTGCGCTGTTTAGACTTGTGGA \\
\hline Pa_mt_Internal & $\mathrm{H}$ & 7 & PaMTIF44 & TGTAAGCCAAAGAGTGAAGAC \\
\hline Pa_mt_Internal & $\mathrm{H}$ & 8 & PaMTIR44 & TTTGGCAGCGGATTTGTTTC \\
\hline Pa_mt_Internal & $\mathrm{H}$ & 9 & PaMTIF46 & ATAGCAATTCCCGCTTGGCTTT \\
\hline Pa_mt_Internal & $\mathrm{H}$ & 10 & PaMTIR46 & TTTAATCCGAAGGGATTAGGAGCTGGT \\
\hline Pa_mt_Internal & $\mathrm{H}$ & 11 & PaMTIF48 & CTGTCATGTAACAAACGACTATCTCC \\
\hline Pa_mt_Internal & $\mathrm{H}$ & 12 & PaMTIR48 & AAAGGGAACCAACAGTGCCAAAC \\
\hline
\end{tabular}


PRIMERNAME PRIMER SEQ

PaMTOF49 GGTTGGCTATAGTTCACCATT

PaMTOR49 ACGGTAAGGTGATAGCAACAA

PaMTIF49 TGGCTATAGTTCACCATTT

PaMTIR49 CGGTAAGGTGATAGCAACAAA

PaMTOF50 TCGATACCCATTGCATTCT

PaMTOR50 TTAAGCGGTTACGTGGTAGTT

PaMTIF50 ACCCATTGCATTCTAATTTCTAC

PaMTIR50 TAAGCGGTTACGTGGTAGTTT

PaMTOF51 TCGATACCCATTGCATTCT

PaMTOR51 CGCTCCCTTTGTCTAGTTAAT

PaMTIF51 CCCATTGCATTCTAATTTCTAC

PaMTIR51 CCCTTTGTCTAGTTAATAAGAGCG

PaZenkOF1 ACTGCCACCAATAACCTACACG

PaZenkOR1 ATCATTGGCACCTGAAAGTTGGT

PaZenkIF1 TGCCACCAATAACCTACACGG

PaZenkIR1 ATTGGCACCTGAAAGTTGGTCTT

PaZenkOF2 TCCAATTTATTCGGCTGCACCA

PaZenkOR2 TTGATCAGCTGGGACTGATAGTT

PaZenkIF2 AATTTATTCGGCTGCACCAACTTTCCC

PaZenkIR2 AGCTGGGACTGATAGTTAGTGTT

PaGapdhOF1 TTTGTCAAGCTGGTTTCCTGGT

PaGapdhOR1 TTCCCATCCACTTCCAGTAAAGA

PaGapdhIF1 TGTCAAGCTGGTTTCCTGGTA

PaGapdhIR1 CCACTTCCAGTAAAGAAAGTAGGAAGCA

PaGapdhOF2 TTATATGTAGACCGCTGCTGAGT

PaGapdhOR2 AGAAACAGAAGTGCTGTCAGGAT

PaGapdhIF2 ATATGTAGACCGCTGCTGAGTC

PaGapdhIR2 AACAGAAGTGCTGTCAGGATTGA 


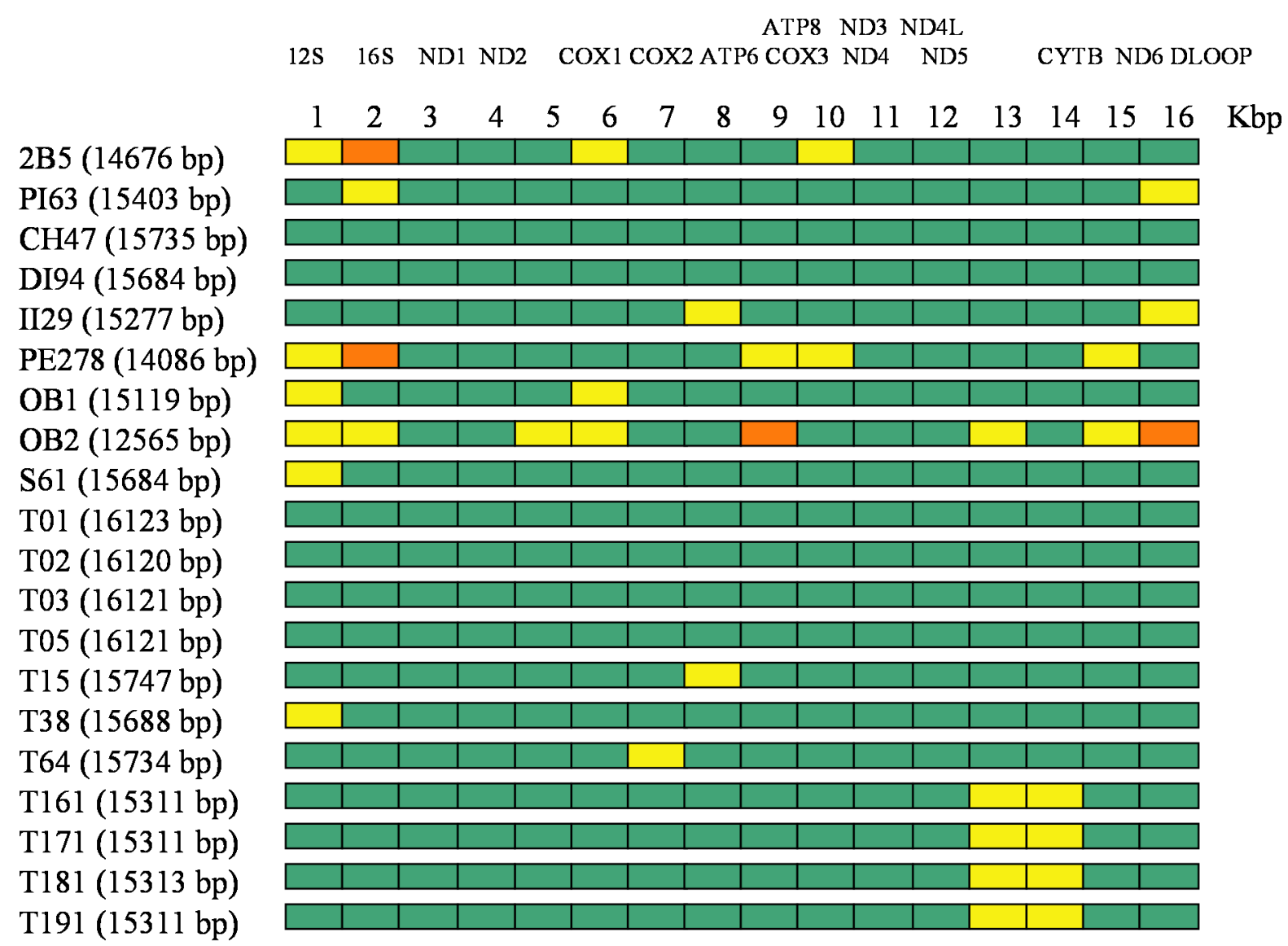

\begin{tabular}{|c|c|}
\hline & Percent sequenced \\
\hline & $50-75 \%$ \\
\hline & $25-50 \%$ \\
\hline
\end{tabular}

2 genomes obtained from individual samples. Total numbers of base pairs recovered are given in parentheses. 


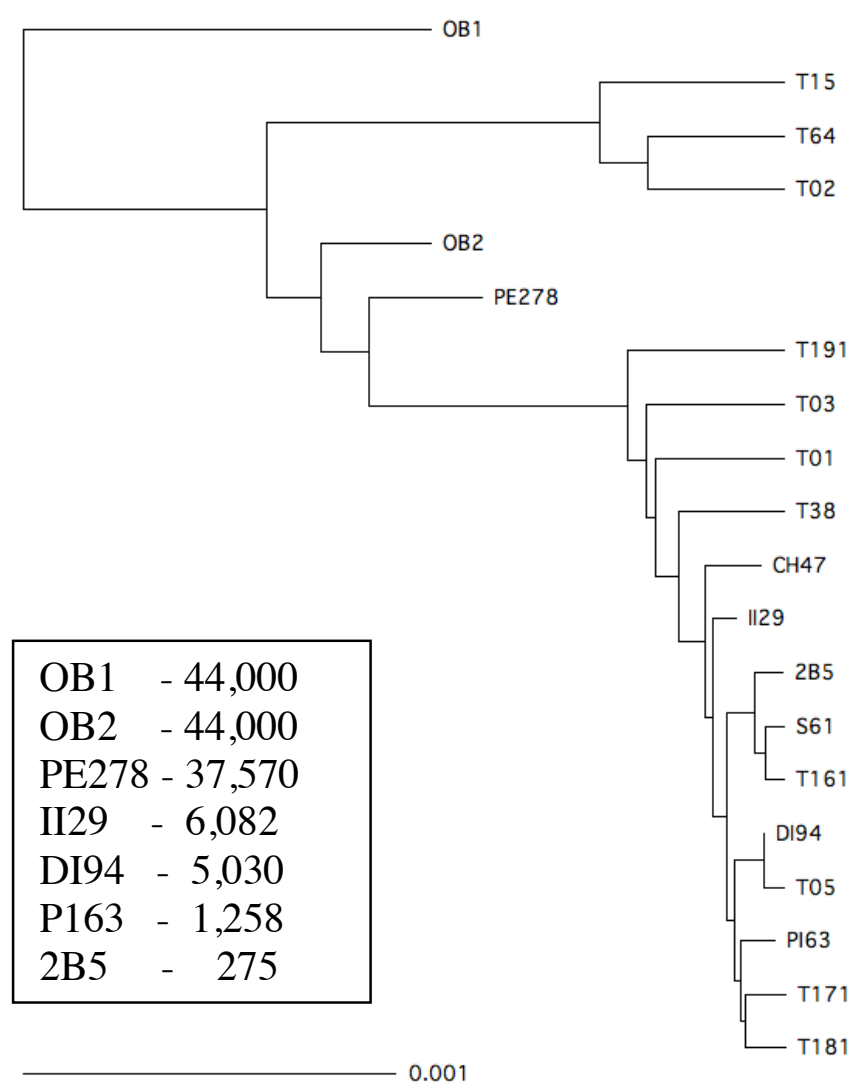

Figure S2. Serial UPGMA tree based on the complete mitogenomes from ancient and modern populations of Adelie penguins. This tree was constructed by using the software Pebble that accounts for the ages of the ancient sequences (Drummond and Rodrigo, 2000). Carbon dated ages of ancient sub-fossil bones are given in the box. 

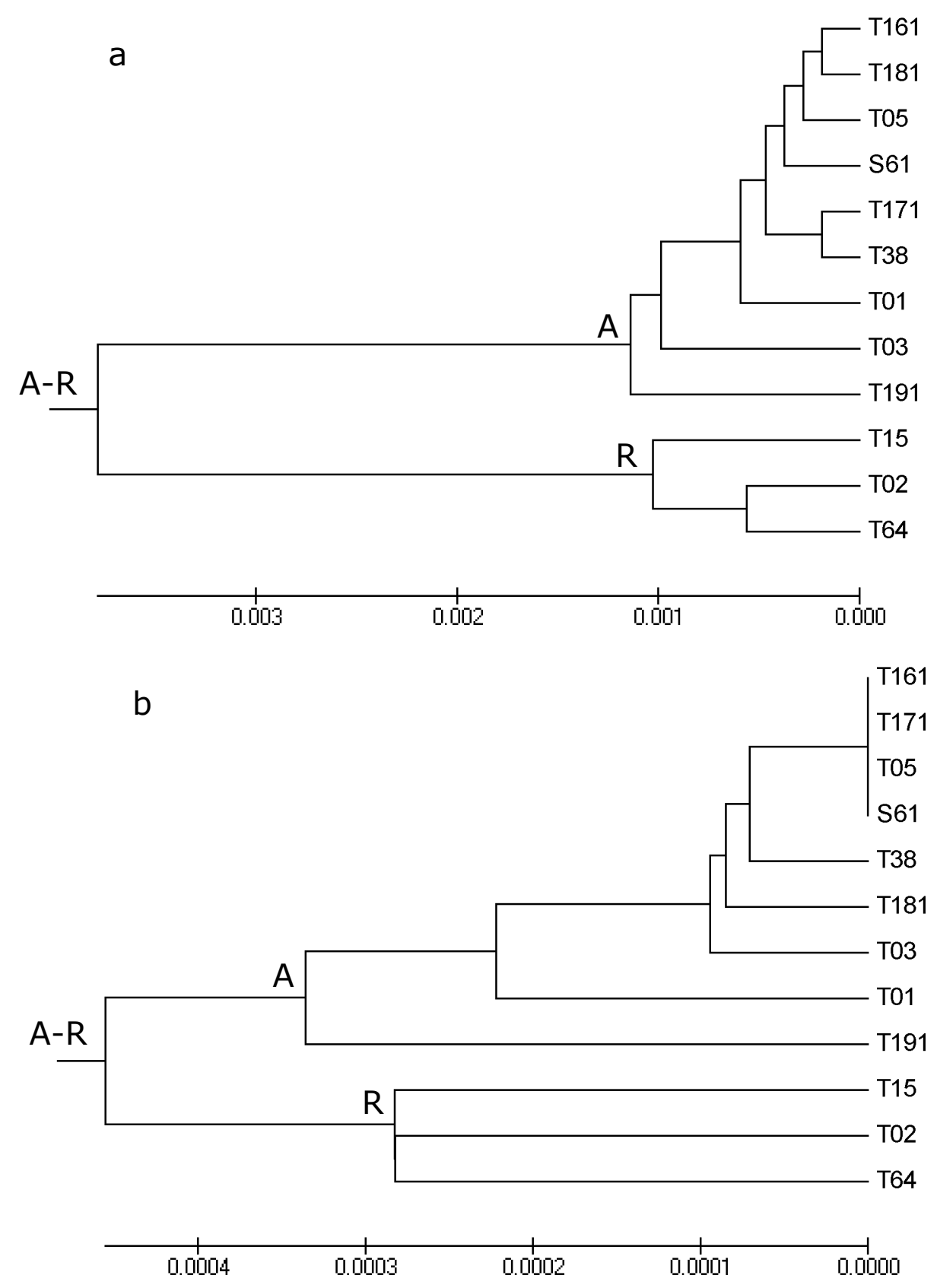

42

43

Figure S3. UPGMA trees of modern Adelie penguins. Trees were constructed using a. synonymous sites b. constrained sites (nonsynomymous sites +tRNAs+rRNAs). The nodes show the two distinct Antarctic (A) and Ross sea (R) lineages, which coalesce at the root (A-R). 


\section{References}

1. Römpler et al. (2006) Multiplex amplification of ancient DNA. Nature Protocols 1: 720-728.

2. Larkin, M., G. et al. (2007). Clustal W and Clustal X version 2.0. Bioinformatics 23: 2947-2948.

3. Tamura, et al. (2007). MEGA4: Molecular Evolutionary Genetics Analysis (MEGA) software version 4.0. Mol Biol Evol 24: 1596-1599.

4. Drummond, A. and Rodrigo, A.G. (2000). Reconstructing genealogies of serial samples under the assumption of a molecular clock using serial-sample UPGMA. Mol Biol Evol 17: 1807-1815.

5. Rambaut, A. (2000). Estimating the rate of molecular evolution: incorporating non-contemporaneous sequences into maximum likelihood phylogenies. Bioinformatics 16, 395-399.

6. Drummond, A. and Rambaut, A. (2007). BEAST: Bayesian evolutionary analysis by sampling trees. BMC Evol Biol 7: 214.

7. Rambaut, A. and Drummond, A.J. (2004). Tracer. University of Oxford. Oxford

8. Yang, Z. (2007). PAML 4: phylogenetic analysis by maximum likelihood. Mol Biol Evol 24: 1586-1591.

9. Rannala, B., and Yang, Z.H. (2003) Bayes estimation of species divergence times and ancestral population sizes using DNA sequences from multiple loci. Genetics 164, 1645-1656 